\title{
Improvement of Dynamic Modeling of Supercapacitor by Residual Charge Effect Estimation
}

\author{
Dimitri Torregrossa, Maryam Bahramipanah, Emil Namor, Rachid Cherkaoui, Senior Member, IEEE, and \\ Mario Paolone, Senior Member, IEEE
}

\begin{abstract}
This paper presents a set of experimental investigations related to the dynamic behavior of supercapacitors (SCs). The experimentally observed results are then used as inputs for the development of an improved version of one of the most common SC $R C$-equivalent circuit models. The key improvement concerns the accurate modeling of the diffusion phenomenon of the SC residual charge during charging/discharging and rest phases. The experimental procedure needed for evaluating the parameters of the proposed model is also given. The accuracy of the obtained model is then experimentally validated for different cycles characterized by different dynamics.
\end{abstract}

Index Terms-Charge redistribution phenomenon, dynamic supercapacitor (SC) behavior, residual charge, SC modeling.

\section{INTRODUCTION}

A S known, supercapacitors [(SCs), also called ultracapacitors], are characterized by high power density, short charging time, and long life duration. These peculiar characteristics make these devices of particular interest in energy-storage applications like fast charging of electric vehicles, medical devices, universal power supply applications, elevators, pulsed laser, etc.

Different representations of the SC behavior in both steady state and dynamic state have been proposed [1]-[11]. They can be grouped in three main categories:

1) time-domain identification leading to interleaved $R C$ equivalent circuit model [1]-[5];

2) frequency-domain identification by impedance spectroscopy model [6]-[9];

3) electrochemical thermal principle identification [10], [11]. The first category allows for inferring in a type of model whose parameters can be easily determined by experimental measurements. However, the physical interpretation of such a category of SC models is not straightforward. As a matter of fact, models [1]-[5] cannot accurately describe the SC voltage behavior

Manuscript received October 24, 2012; revised January 24, 2013 and March 13, 2013; accepted March 29, 2013. Date of publication April 24, 2013; date of current version August 23, 2013.

D. Torregrossa, M. Bahramipanah, R. Cherkaoui, and M. Paolone are with the Distributed Electrical Systems Laboratory, Swiss Federal Institute of Technology (EPFL), 1015 Lausanne, Switzerland.

E. Namor is with the University of Padova, 35137 Padova, Italy.

Color versions of one or more of the figures in this paper are available online at http://ieeexplore.ieee.org.

Digital Object Identifier 10.1109/TIE.2013.2259780 during high-dynamic current profiles where the redistribution of residual charge stored in the SC can play a key role.

The second category allows for obtaining a frequencydomain model of SCs by specific experimental investigations based on the use of network analyzers or high-bandwidth (6 kHz) impedance measurement systems (electrochemical impedance spectroscopy) [5], [6].

The third category of models is the more general as it can be applied to general electrochemical storage systems like SCs and batteries. In particular, as discussed in [10], this method defines large-signal time-dependent model of general electrochemical storage systems by using the Poisson-Nernst-Planck electrodiffusion theory suitably combined with the analysis of physical attributes of the system (e.g., nonlinear polarization of bulk electrolyte reactions, changes of transport coefficients, etc.).

With reference to SC modeling, the common drawback among the aforementioned models is that they do not focus on some elementary phenomena that take place during the SC normal operation such as the redistribution of the so-called "residual charge" during the following: 1) the SC charging and 2) the SC relaxation phase. In Section II, the aforementioned phenomena are illustrated in order to provide the added value of the model proposed in this paper.

As is known, the complete extraction of the SC charges requires very long times (i.e., on the order of several days). As a consequence, there is always an amount of charges that remains stored inside the SC. Even after partial SC discharges, the amount of the SC-stored charges plays a key role in the subsequent SC behavior. This specific drawback has, indeed, motivated this study in order to develop a new SC model. In what follows, the term "residual charge" $-Q_{R}-$ defines the amount of the remaining SC charge stored into the device after a discharge that has resulted into a specific value of the $\mathrm{SC}$ voltage $\left(V_{\mathrm{SC}}\right)$ achieved at the end of the discharge. An SC during its normal operation is charged and discharged at different voltages with different values of current. These phases can have long or short durations and have nonnegligible effects on the SC dynamic. For different SCs sizes, it could be experimentally observed that each cycle changes its dynamic behavior. Two main effects due to the SC cycling can be observed: the first concerns the duration of the charging phase, and the other refers to the redistribution phase. In particular, after the first SC charging-discharging cycle, there is a remaining $Q_{R}$ stored into the SC that cannot be easily extracted. 


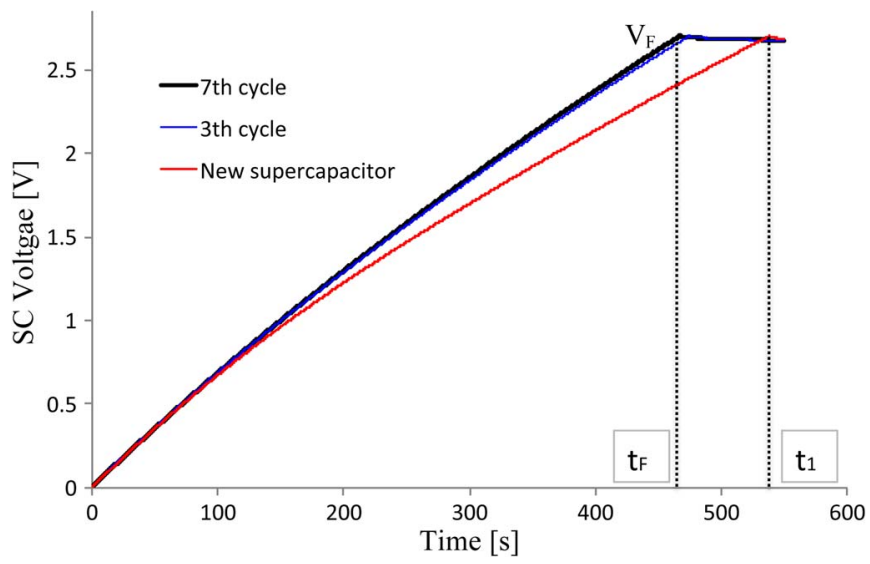

Fig. 1. Time evolution of the supercap voltage for different charge-discharge cycles.

As expected, the presence of $Q_{R}$ reduces considerably the charging time needed to reach a certain voltage value in comparison with the time needed when the targeted SC was without any residual charge. Within this context, this paper focuses on the electrical SC modeling during dynamic cycles by properly taking into account the influence of $Q_{R}$ on the SC dynamic behavior.

This paper is structured as follows. Section II focuses on the experimental evidences related to the $Q_{R}$ effects on the $\mathrm{SC}$ behavior. Then, in Section III, a brief state of the art of the different SC models is presented along with a discussion of their advantages versus drawbacks. Section IV presents the proposed SC model along with the procedures for evaluating its parameters. Section V presents the experimental validation of the proposed model by making reference to dynamic cycles including both short and long time durations of charging and redistribution phases. Conclusions and final remarks concerning the contribution of the proposed model are given in the last section.

\section{EXPERIMENTAL EVIDENCES OF SC CHARGE REDISTRIBUTION}

Fig. 1 shows the time evolution of the voltages across a $325-\mathrm{F} \mathrm{SC}$ to reach its nominal voltage $(2.7 \mathrm{~V})$ when charged with 2 A. $^{1}$ Each curve refers to a charge, subsequent to a discharge that has lasted to reach an SC voltage $V_{\mathrm{SC}}=50 \mathrm{mV}$. This figure shows that, even with identical SC final discharge voltage (i.e., $50 \mathrm{mV}$ ), the subsequent $\mathrm{SC}$ charging curves exhibit different behaviors (i.e., differences on the order of several tens of seconds) indicating, therefore, different values of $Q_{R}$ (i.e., remaining SC charges in correspondence of an $\mathrm{SC}$ voltage of $50 \mathrm{mV}$ ). This indicates a progressive increase of the $Q_{R}$ that reaches, for the targeted $360-\mathrm{F} \mathrm{SC}$, its maximum value after seven cycles. The time needed to reach $2.7 \mathrm{~V}$ is of $550 \mathrm{~s}\left(t_{1}\right.$ in Fig. 1) when the SC has no residual charge. When the SC has been cycled seven times, this time is equal to $470 \mathrm{~s}\left(t_{F}\right.$ in Fig. 1).

\footnotetext{
${ }^{1}$ All the experimental tests on the SC have been carried out into a dedicated climatic chamber at a constant temperature of $25^{\circ} \mathrm{C}$.
}

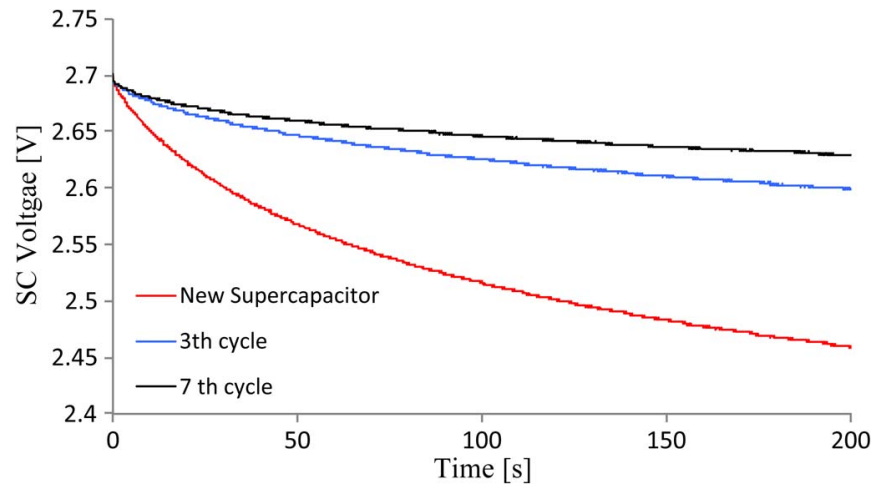

Fig. 2. Time evolution of the SC voltage during rest phase for different values of residual charge.

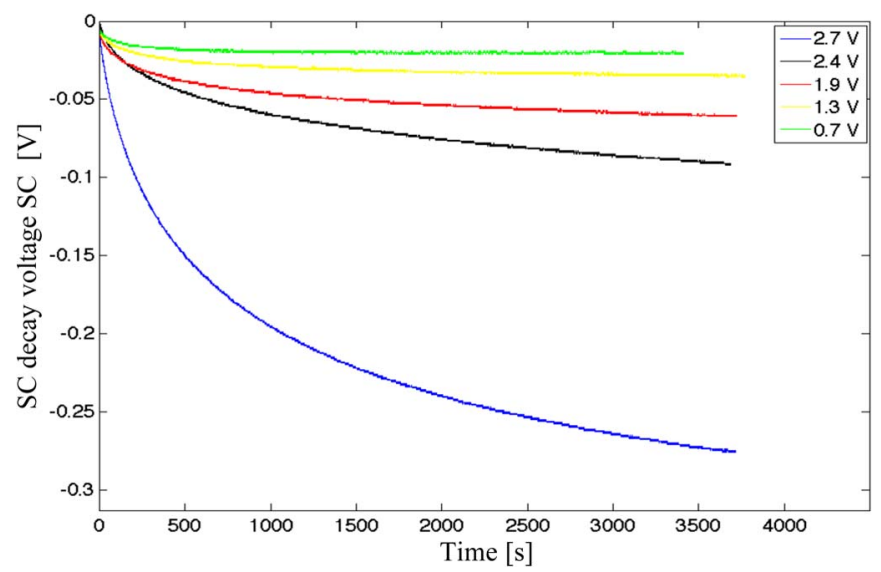

Fig. 3. Time evolution of the SC voltage during rest phase for different final voltage values.

The physical explanation of this behavior is based on the fact that, during each charging phase, the amount of $Q_{R}$ stored inside the SC macropores available at the electrode-electrolyte interface (i.e., those more easily accessible by charges) increases, and consequently, it participates actively to the subsequent charging phase. Once the availability of macropores is saturated (for the targeted SC, this happens after seven complete cycles), the $Q_{R}$ reaches its maximum value.

Fig. 2 shows the second main effect of $Q_{R}$. The time to reach the $\mathrm{SC}$ open-circuit voltage $\left(V_{\mathrm{OC}}\right)$, after the complete redistribution phenomenon, as well as the value $V_{\mathrm{OC}}$ itself, depends strongly on the $Q_{R}$. The higher the $Q_{R}$, the higher the $V_{\mathrm{OC}}$ and the lower the time needed to reach it. As shown in Fig. 3, a different time evolution of the so-called decay voltage across the SC can be observed for different final voltage values ( $V_{F}$ in Fig. 2 ). In order to highlight the SC decay-voltage dynamic, Fig. 3 shows the difference between the $\mathrm{SC}$ voltage and the $V_{F}$.

As is known, the SC redistribution phenomenon is the process of homogenization of the charges on the whole SC electrode surfaces during the relaxation phase. It takes a long time to be completed (typical time constants are on the order of $700-800$ s). It is well known that the redistribution phenomenon is caused by the irregularity of the SC electrode porous carbon structure involving a gradient of ion concentration within the SC electrolyte. This gradient depends on the different 
time constants of the macro-, meso-, and micropores of the electrode-electrolyte interface [12]. This means that, during the charging phase, the early-time charges migrate easily toward the macropore and then, during the relaxation phase, toward the other smaller pores.

The voltage drop process of an SC in rest phase is described in detail in [15] where the physical mechanisms governing this phenomenon are given. In summary, in [15], it is found that, after the SC is disconnected from the charging circuit, the excess ionic concentration near the carbon-to-electrolyte surface will follow a diffusion mechanism. Part of these ions will diffuse into the electrolyte, while some others will diffuse toward the carbon surface. The last involve a reduction of the number of excess charges in the carbon itself and, therefore, will produce a decrease of the SC open-circuit voltage.

As it will be clarified in Sections IV-C and V-A (Figs. 7 and 10 ), the quantity of residual charges redistributing during the charging and relaxation phases depends on the $V_{\mathrm{OC}}$. In this respect, along with the explanations already given for Fig. 1, it is possible to physically sustain what is shown by Figs. 2 and 3. Concerning Fig. 2, it is clear that, after each charging phase, the amount of $Q_{R}$ increases. This means a higher amount of charges stored in the aforementioned macropores and a consequent larger gradient of charges. Such a behavior involves a faster and more important redistribution phenomena during the relaxation phase with a mitigation of the $\mathrm{SC}$ voltage decay (after each cycle, a little quantity of charges is stored in the medium and micropores as well).

Fig. 3 concerns the SC voltage time evolution during a rest phase for different final voltage values once the targeted SC has been already cycled in order to saturate the macropores.

Additionally, it should be underlined that the charges participating to the redistribution phenomena are those already stored inside the targeted SC, namely, $Q_{R}$, plus some other charges injected by the power source to the SC during the charging phase. If the charging phase is stopped before reaching the nominal voltage value, the total quantity of charges is less than the one at nominal voltage. The gradient charge will be lower and the voltage decay as well. Otherwise, if the SC reaches its nominal voltage value, the charge gradient will have a higher value and, as a consequence, will involve longer relaxation phase along with higher voltage decay. It is worth noting that these considerations are true if the targeted SC has been already cycled.

From this standpoint, it is important to evaluate the SC initial condition in order to estimate the amount of the $Q_{R}$ and properly include it into a suitable SC model.

Table I summarizes the values of $V_{\mathrm{OC}}$ subsequent to different values of voltage reached during the charging phase.

As it will be illustrated next, if the two aforementioned effects are not considered, the final voltage $V_{F}$, as well as the whole SC voltage profile during a dynamic cycle evaluated by available models, can largely differ from measured values. In this respect, in [9], the importance of the charge movement within the SCs has been already observed.

It should be noticed that the different voltage dynamic behaviors, shown in both Figs. 2 and 3, start to be nonnegligible from $150 \mathrm{~s}$ after the turnoff of the SC supply.
TABLE I

Relation Between Reached Final Voltage $V_{F}$ AND $V_{\text {OC }}$

\begin{tabular}{|c|c|}
\hline$V_{F}(\mathrm{~V})$ & $V_{O C}(\mathrm{~V})$ \\
\hline 2.7 & 2.46 \\
\hline 2.4 & 2.13 \\
\hline 2.1 & 1.81 \\
\hline 1.8 & 1.49 \\
\hline 1.5 & 1.23 \\
\hline 1.2 & 0.97 \\
\hline 0.9 & 0.71 \\
\hline 0.6 & 0.48 \\
\hline 0.3 & 0.22 \\
\hline
\end{tabular}

To the best of the authors' knowledge, few papers have focused on the estimation of the SC $Q_{R}$ (e.g., [13] and [14]). However, none of them have used this information to infer an updated SC model.

\section{Comparative AnAlysis And Limits OF EXISTING SC MODELS}

The majority of the SC models based on interleaved $R C$ equivalent circuits are presented in [1] and [2]. These models are composed by three main lumped-parameter branches. As clearly explained in the literature (e.g., [1] and [2]), the first branch aims at representing the SC behavior when it is connected to an external source or load; the second and the third branches represent the medium and long time duration of the redistribution phenomenon, respectively. As already stated, the main limitation of [1] and [2] is that they do not take into account the $Q_{R}$. Such a limitation has an influence on the assessment of the SC model parameters as well as on the SC model applicability. Additionally, the experimental validation proposed in [1] and [2] is carried out without providing an accuracy assessment of the obtained model versus the SC real dynamic behavior. Furthermore, as clearly stated in [2], their model is not accurate for low voltages (i.e., below $1 \mathrm{~V}$ ) and for long time estimation of the SC $V_{\mathrm{OC}}$. Similar considerations can be done for the work presented in [8].

Concerning the model presented in [3], it has been validated by experimental results for two different current profiles and not for the whole nominal voltage range $(0-2.7 \mathrm{~V})$. In this respect, any performance of the proposed model during a dynamic cycle is presented. Similar considerations can be done for [5]-[7]. In particular, in [5], the authors analyzed the impact of the increasing number of $R C$ branches on the accuracy of the whole model, and they concluded that the six-branch model is the most accurate for modeling the self-discharge. However, the accuracy of the proposed model for dynamic cycle with long charging/discharging and redistribution phases has not been illustrated.

Concerning [9], the different redistribution behaviors of the $\mathrm{SC}$ have been modeled by considering a parameter variation of the model itself. However, [5]-[9] did not show the accuracy of the model during slow charge and discharge phases as well as during long redistribution phases and dynamic cycles. 


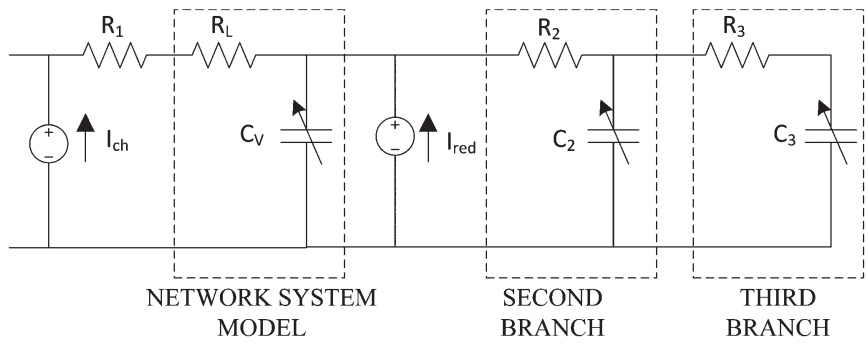

Fig. 4. Proposed SC model.

The most thorough investigation of the behavior of an SC during long time observation has been done in [4]. In this paper, experimental investigations have driven the conclusion about the false assumption, done in several works, for example, in [5], that the voltage drop observed in SCs after their disconnection to a dc source is due to their self-discharge originated by a charge-leakage phenomenon. We have also experimentally observed the findings of [4], namely, if the SC is charged for a longer time (i.e., we reach the same $V_{F}$ with lower supply currents), higher $Q_{R}$ values are obtained.

The only work showing some results about the impact of the charge history on SCs behavior is [12]. In this paper, the authors observed a different relative decay depending on the value of final voltage reached during the charging phase. However, they did not give a physical explanation of this behavior, and it is not clear how their model can take it into account.

\section{Proposed SC Model}

The model proposed in this paper is an improved version of the model presented in [1] and [2].

The main goal of the proposed model is to accurately predict the SC behavior when it is cycled with long time dynamics. Fig. 4 illustrates the new proposed model, where $R_{1}$ is the SC input electrode resistance, $R_{L}$ and $C_{V}$ are the resistance and the nonlinear capacitance of the so-called "SC network system model" (see [1] and [2]), $R_{2}$ and $C_{2}$ are the resistance and the nonlinear capacitance of the SC second branch, and $R_{3}$ and $C_{3}$ are the resistance and the nonlinear capacitance of the third SC branch.

As it can be observed in the proposed model shown by Fig. 4, two current sources, $I_{\mathrm{CH}}$ and $I_{\mathrm{RED}}$, are included. These two devices allow for improving the SC dynamics by taking into account the diffusion of the residual charge during charge/discharge phases and redistribution one, respectively. The determination of the parameters of these two current sources, as well as the demonstration of their neutrality about the SC charge preservation, will be discussed next.

\section{A. Optimal Assessment of Standard SC Branch Parameters}

The first parameter, namely, the SC input electrode resistance $R_{1}$, is evaluated by measuring the voltage drop across the SC terminals when a step current is applied. ${ }^{2}$

\footnotetext{
${ }^{2}$ The estimation of this parameter can be done with arbitrary current amplitudes; however, we have adopted the SC rated value.
}

The current generators $I_{\mathrm{CH}}$ and $I_{\mathrm{RED}}$ account for the diffusion of the $Q_{R}$; they are inherently inactive when the SC is charged for the first time. In such a specific condition, by making reference to the model shown in Fig. 4, the SC terminal voltage $v_{\mathrm{SC}}(t)$ can be represented only as a function of the SC current and the relevant parameters, as follows:

$$
v_{\mathrm{SC}}(t)=f_{1}\left[i(t), R_{L}, C_{V i}\right]+F_{2}\left[i(t), R_{2}, C_{2 i}\right]+F_{3}\left[i(t), R_{3}, C_{3 i}\right]
$$

where

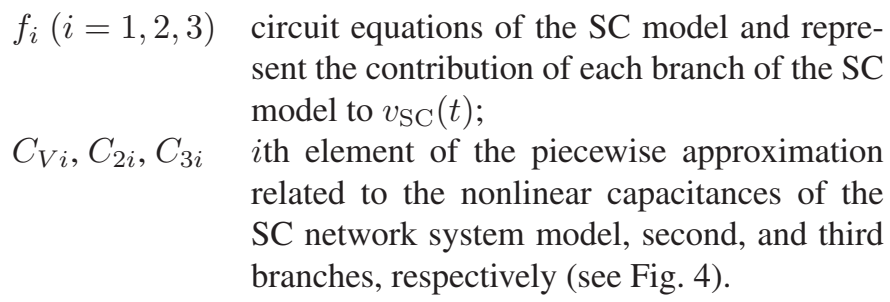

The evaluation of the parameters of (1) has been performed by following a modified procedure proposed in [1] and [2]. In particular, the variation of the SC voltage has been divided into a certain number of intervals (in our case, ten) between zero and the SC rated voltage (i.e., 0-2.7 V). For each of these intervals, a first set of the SC parameters has been evaluated by using the procedure given in [1] and [2]. Then, in order to improve the values of these parameters, a least square fitting procedure has been used. This last procedure makes use of the measured SC voltage $v_{\mathrm{SC}, M}(t)$, during a charging and a redistribution phases. Indeed, for each of these phases, we can assume that $v_{\mathrm{SC}}(t)$ essentially depends on the SC network system model and on the second/third branch, respectively. In other words

$$
\begin{aligned}
v_{\mathrm{SC}}^{\text {charge }}(t) & =F_{1}\left[i(t), R_{2}, C_{2 V i}\right] \\
v_{\mathrm{SC}}^{\mathrm{RED}}(t) & =F_{2}\left[i(t), R_{2}, C_{2 i}\right]+F_{3}\left[i(t), R_{3} C_{3 i}\right] .
\end{aligned}
$$

It is therefore possible to define the following optimal problems for inferring the model parameters:

$$
\begin{gathered}
\left.\underset{R_{L}, C_{V i}}{\arg \min _{\mathrm{SC}, M}}\{t)-f_{1}\left[i(t), R_{L}, C_{V i}\right]\right\}^{2} \\
\arg \min _{R_{2}, C_{2 i}, C_{3 i}}\left\{\begin{array}{l}
v_{\mathrm{SC}, M}^{\mathrm{RED}}(t)-f_{2}\left[i(t), R_{2}, C_{2 i}+\right. \\
-F_{3}\left[i(t), R_{3}, C_{3 i}\right]
\end{array}\right\}^{2} .
\end{gathered}
$$

It is worth noting that the optimal problem formulated in (4) makes use of the parameters that have been obtained by the solution of (3).

The aforementioned optimal assessment of the SC parameters has a nonnegligible influence on the $v_{\mathrm{SC}}(t)$. Indeed, Fig. 5 illustrates the comparison between the measurement and the simulation results coming from the model where the parameters have been assessed with the aforementioned optimal procedure versus the approach proposed in [1] and [2]. The mean-square errors between the measurements and the $v_{\mathrm{SC}}(t)$ provided by the SC model with the parameters assessed by using the two procedures are as follows: 0.0044 for Zubieta's model [2] and near null for the model proposed here.

It should be underlined that the parameters of the model presented in this section allow for an accurate prediction of the $\mathrm{SC}$ voltage if the targeted SC has not been charged before (or if 


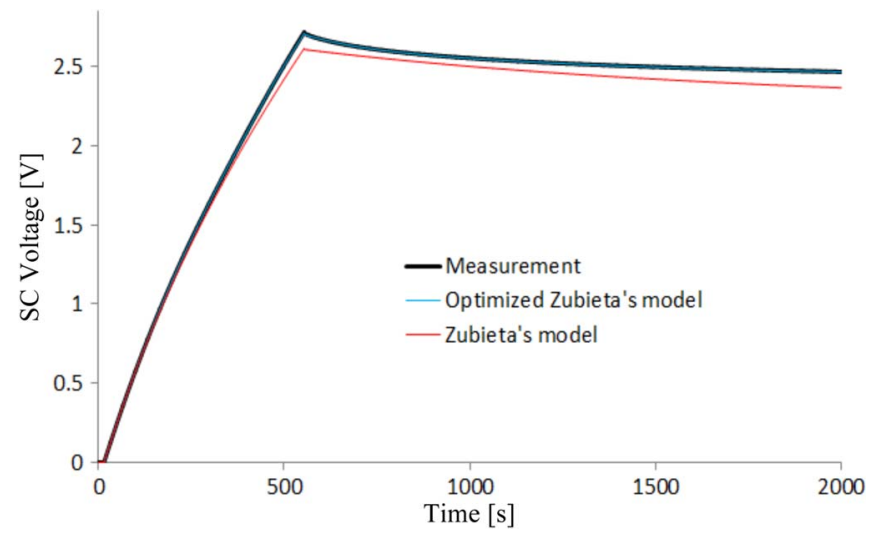

Fig. 5. Experimental validation of the SC model without any residual charge.

TABLE II

Resistance, Time Constant, and Charge Ratio Values OF THE TARGETED 325 F SC MODEL

\begin{tabular}{|c|c|}
\hline$R_{1}$ & $2.5 \mathrm{~m} \Omega$ \\
\hline$R_{L}$ & $3.7 \mathrm{~m} \Omega$ \\
\hline$R_{2}$ & $2.3 \Omega$ \\
\hline$R_{3}$ & $18.71 \Omega$ \\
\hline$\tau$ & $1000 \mathrm{~s}$ \\
\hline$\eta_{2}$ & 0.3 \\
\hline$\eta_{3}$ & 0.7 \\
\hline
\end{tabular}

TABLE III

First Nonlinear Capacitance Values (F)

\begin{tabular}{|c|c|}
\hline Range voltage value $(\mathrm{V})$ & Capacitance value \\
\hline $0-0.3$ & 278.1 \\
\hline $0.3-0.6$ & 281.3 \\
\hline $0.6-0.9$ & 288.2 \\
\hline $0.9-1.2$ & 297 \\
\hline $1.2-1.5$ & 307 \\
\hline $1.5-1.8$ & 318 \\
\hline $1.8-2.1$ & 329 \\
\hline $2.1-2.4$ & 337 \\
\hline $2.4-2.7$ & 348 \\
\hline Over 2.7 & 349 \\
\hline
\end{tabular}

TABLE IV

Second Nonlinear Capacitance Values (F)

\begin{tabular}{|c|c|}
\hline Range voltage value $(\mathrm{V})$ & Capacitance value \\
\hline $0-0.3$ & 54.3 \\
\hline $0.3-0.6$ & 54.4 \\
\hline $0.6-0.9$ & 54.9 \\
\hline $0.9-1.2$ & 56.3 \\
\hline $1.2-1.5$ & 58 \\
\hline $1.5-1.8$ & 60.1 \\
\hline $1.8-2.1$ & 62.1 \\
\hline $2.1-2.4$ & 64.2 \\
\hline $2.4-2.7$ & 65.8 \\
\hline Over 2.7 & 68 \\
\hline
\end{tabular}

it has been completely discharged before the test). As discussed in Section III, if the SC has a residual charge, its behavior is totally different. Tables II-V illustrate the value of the model parameters.
TABLE V

Third Nonlinear Capacitance Values (F)

\begin{tabular}{|c|c|}
\hline Range voltage value $(V)$ & Capacitance value \\
\hline $0-0.3$ & 16.9 \\
\hline $0.3-0.6$ & 16.9 \\
\hline $0.6-0.9$ & 17.1 \\
\hline $0.9-1.2$ & 17.5 \\
\hline $1.2-1.5$ & 18 \\
\hline $1.5-1.8$ & 18.7 \\
\hline $1.8-2.1$ & 19.3 \\
\hline $2.1-2.4$ & 20 \\
\hline $2.4-2.7$ & 21.2 \\
\hline Over 2.7 & 21.2 \\
\hline
\end{tabular}

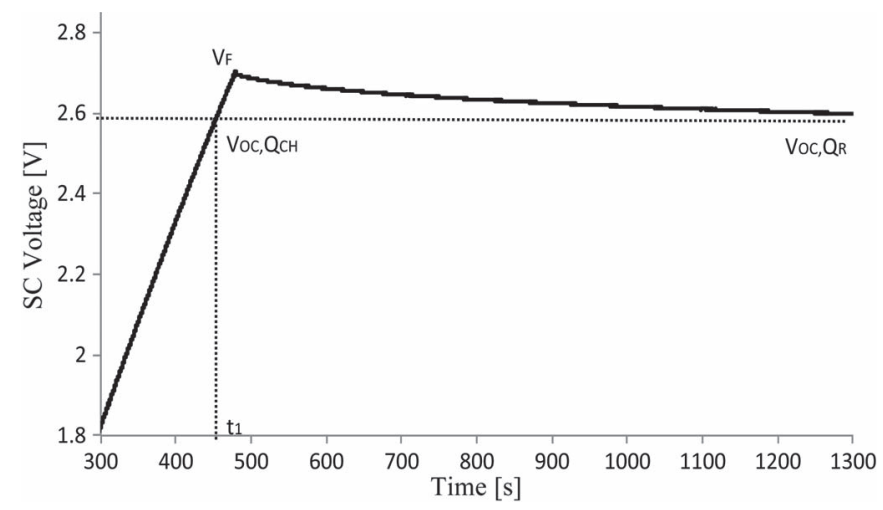

Fig. 6. Example of the difference of residual charge.

\section{B. Current Sources for the Representation of $Q_{R}$ Diffusion}

Let us consider an SC that is charged to reach a predefined $V_{F}$ and, subsequently, disconnected from the source in order to reach its equilibrium. For $t \rightarrow \infty$, we have that $v_{\mathrm{SC}}(t) \rightarrow$ $V_{\mathrm{OC}}$. After the $\mathrm{SC}$ equilibrium, the device is discharged to a null value of SC voltage, and therefore, we can associate to the $V_{\mathrm{OC}}$ a relevant value of charge that, in view of what is illustrated earlier, is the $Q_{R}$. The phenomenon that can be observed (Fig. 6) is that, during the SC charging, we can associate an amount of charge $Q_{\mathrm{CH}}$ (at time $t_{1}$ ) given to the SC in correspondence of the SC voltage equal to $V_{\mathrm{OC}}$ (see Fig. 6). The difference between $Q_{R}$ and $Q_{\mathrm{CH}}$, henceforth called $\Delta Q$, represents an amount of charge that will be used in what follows to define $I_{\mathrm{CH}}$ and $I_{\mathrm{RED}}$. The nonlinear link between $V_{\mathrm{OC}}$ and $\Delta Q$ is illustrated in Fig. 10.

A possible physical explanation of $\Delta Q$ is the following: It represents the amount of charge that the SC stores, during the charging phase, with the need of higher potential differences. With larger times, the diffusion phenomenon of this charge takes place with a specific time constant together with the displacement of preavailable SC charges. Therefore, it is possible to represent this phenomenon by using a specific current generator $I_{\mathrm{CH}}$ that is active only when the $\mathrm{SC}$ is connected to a source, or a load, in order to deliver the $\Delta Q$ amount

$$
i_{\mathrm{CH}}(t)= \pm \frac{\Delta Q}{\tau} e^{-\frac{t}{\tau}}
$$

where $\tau$ is the time constant of the redistribution of $\Delta Q$. The value of this time constant depends on the size of the targeted SC (it depends on the macro-meso-microstructure of the electrode-electrolyte interface) and, therefore, has been 


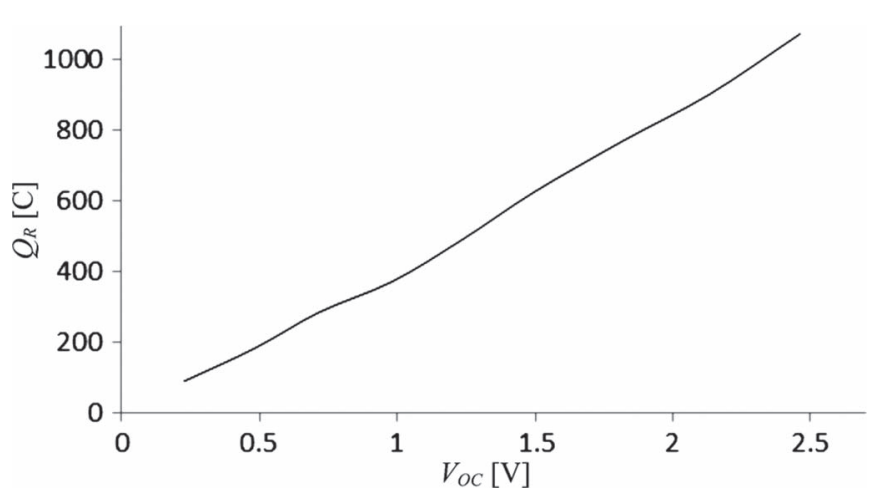

Fig. 7. Residual charge and open-circuit voltage.

experimentally determined and equals $1000 \mathrm{~s}$. The current will be positive if we are considering a charging phase and will be negative vice versa. It is worth noting that this generator does not violate the charge balance of the SC since it is not injecting an external current but it models the movement of the charges already stored in the macropores. The time integral of this current has been verified not to be higher than $\Delta Q$.

The generator $I_{\mathrm{RED}}$, which is active only during the redistribution phases, should deliver a current following this equation:

$$
i_{\mathrm{RED}}(t)= \pm\left(\eta_{2} \frac{\Delta Q_{\mathrm{RED}}}{\tau_{2}}+\eta_{3} \frac{\Delta Q_{\mathrm{RED}}}{\tau_{3}} e^{-} \frac{t}{\tau_{3}}\right)
$$

where $\Delta Q_{\mathrm{RED}}$ is the amount of the residual charge available for the redistribution phase. This value can be analytically calculated as

$$
\Delta Q_{\mathrm{RED}}=\Delta Q-\int_{a}^{t_{\mathrm{ch}}} i_{\mathrm{CH}}(t) d t
$$

where $t_{\mathrm{ch}}$ is the duration of the charging phase.

The $\Delta Q_{\mathrm{RED}}$ should be delivered by following the two time constants associated to the dynamics of the redistribution phenomena. The time constant $\tau_{2}$ is related to the diffusion of the charges through the medium sizes of the electrolyte pores, and $\tau_{3}$ is related to the micropores. Parameters $\eta_{2}$ and $\eta_{3}$ are the quota of $\Delta Q_{\mathrm{RED}}$ to be redistributed with the time constant $\tau_{2}$ and $\tau_{3}$, respectively. The current will be positive if we are considering a redistribution phase just after a charging phase, and it will be negative if we are considering a redistribution phase just after a discharging phase.

It is worth noting that the input of the proposed model are only as follows: 1) the value of the open-circuit voltage before any utilization of the targeted SC and 2) the input current provided by a voltage or current source. Therefore, the proposed model is valid irrespective of the type of adopted source/load applied to the SC.

\section{Determination of $\Delta Q$}

First, the relationship between $V_{\mathrm{OC}}$ and $Q_{R}$ has been assessed (see Fig. 7).

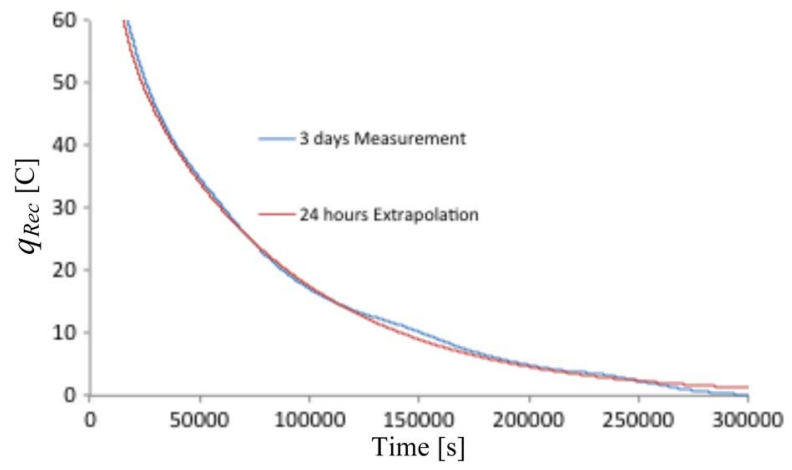

Fig. 8. Comparison between the extrapolated residual charge and the threeday measurement.

The evaluation of the $V_{\mathrm{OC}}$ and $Q_{R}$ has been performed by using the following procedure.

1) From a zero-voltage condition, after a complete SC discharge $\left(V_{\mathrm{OC}}=0\right)$, the $\mathrm{SC}$ has been charged until the required voltage (the charge delivered by the generator has been measured).

2) The supply generator has been turned off, and the $v_{\mathrm{SC}}(t)$ has been recorded during $1 \mathrm{~h}$; the final value of $v_{\mathrm{SC}}(t)$ measured after $1 \mathrm{~h}$ provides a first estimation of the $V_{\mathrm{OC}}$.

3) Then, the SC has been discharged for $1 \mathrm{~h}$ with a simple variable resistor and then short-circuited for $24 \mathrm{~h}$.

4) The time evolution of the charge extracted from the phase described in (c) has been recorded and named as $q_{\mathrm{REC}}(t)$. The final value of $q_{\mathrm{REC}}(t)$, measured after $1 \mathrm{~h}$, provides a first estimation of $Q_{R}$ for the measured $V_{\mathrm{OC}}$.

5) The real $V_{\mathrm{OC}}$ and $Q_{R}$ have been calculated by an exponential fitting provided by

$$
\begin{aligned}
q_{\mathrm{rec}}(t) & =A e^{\lambda_{1} t}+B e^{\lambda_{2} t} \\
v_{\mathrm{oc}}(t) & =C e^{\lambda_{3} t}+D e^{\lambda_{4} t} .
\end{aligned}
$$

The constants $A, B, C, D, \lambda_{1}, \lambda_{2}, \lambda_{3}$, and $\lambda_{4}$ have been determined after the exponential fitting, and from this standpoint, (8) and (9) allow for evaluating the charge and the open-circuit voltage for long time estimation. The constant $A$ equals 0.3 , and $B$ equals 0.7. These two values are used to evaluate the parameters $\eta_{2}=A$ and $\eta_{3}=B$.

These extrapolations have been done for evaluating the $V_{\mathrm{OC}}$ after $5 \mathrm{~h}$ and the $Q_{R}$ after three days. In order to validate the robustness of this extrapolation, Fig. 8 illustrates the comparison of the extrapolated value of the residual charge (by using the data coming from the first $24 \mathrm{~h}$ ) and the measurement performed during three days.

Then, the targeted SC has been cycled seven times with complete charging phase and discharging phase (until $v_{\mathrm{SC}}(t)$ reaches $50 \mathrm{mV}$ ). After that, the $\mathrm{SC}$ has been charged until its nominal voltage value $(2.7 \mathrm{~V})$ and completely discharged through several redistribution phases as shown in Fig. 9. For this figure, the same considerations as those of Fig. 6 can be done. For each value of $V_{\mathrm{OC}}$, the residual charge has been measured. The difference between this value and that one at the same value of voltage during the charging phase has been recorded (as already illustrated in Fig. 6). Fig. 10 illustrates the 

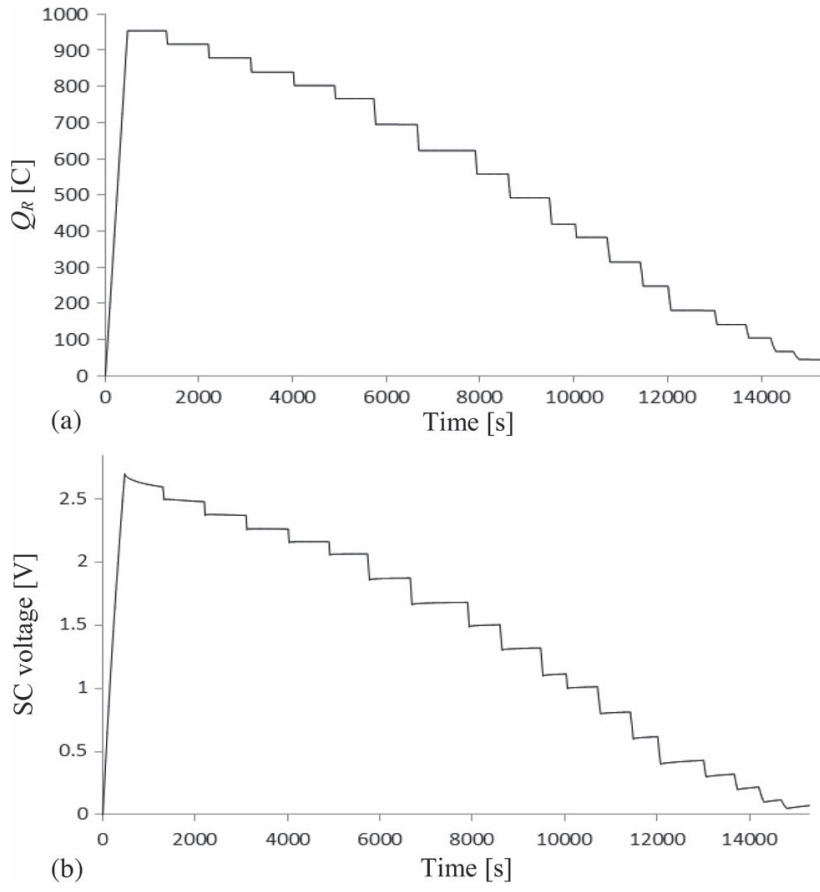

Fig. 9. Time evolution of the (a) $Q_{R}$ and (b) $v_{\mathrm{SC}}$ for the determination of the difference of residual charge.

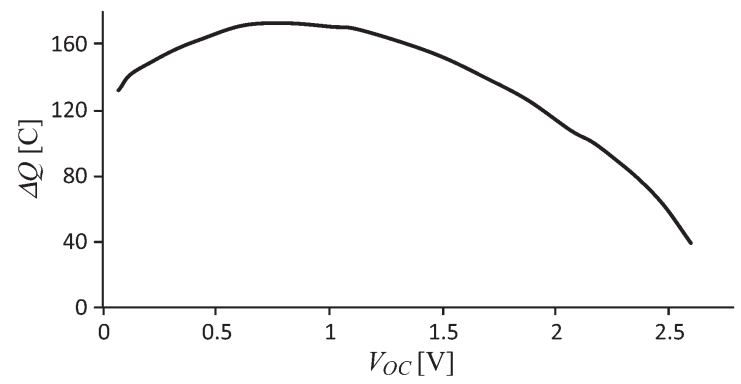

Fig. 10. Difference of residual charge for different values of $V_{\mathrm{OC}}$.

relationship between this difference in the residual charge for each possible value of $V_{\mathrm{OC}}$. It should be underlined that, for each point of Fig. 10, the difference between $Q_{R}$ and $Q_{\mathrm{CH}}$, the residual charge $Q_{R}$ corresponding to the initial voltage equal to $50 \mathrm{mV}$ (the SC is not complete discharged) has been added.

\section{EXPERIMENTAL VALIDATION}

In this section, the experimental assessments of the proposed parameters model are detailed. Such an assessment has also allowed the validation of the proposed model. The experimental investigations have been carried out by using a specific test bench where SC charging/discharging can be automatically controlled. The SC voltage and current have been directly measured without any signal conditioning by using the following National Instruments A/D conversion cards: 1) C-series 9215 $\pm 10 \mathrm{~V}, 16$-b voltage digitizer operating at the maximum sampling frequency of $100 \mathrm{kHz}$ and 2) C-series $9227 \pm 5 \mathrm{~A}, 24-\mathrm{b}$ current digitizer operating at the maximum sampling frequency of $50 \mathrm{kHz}$.

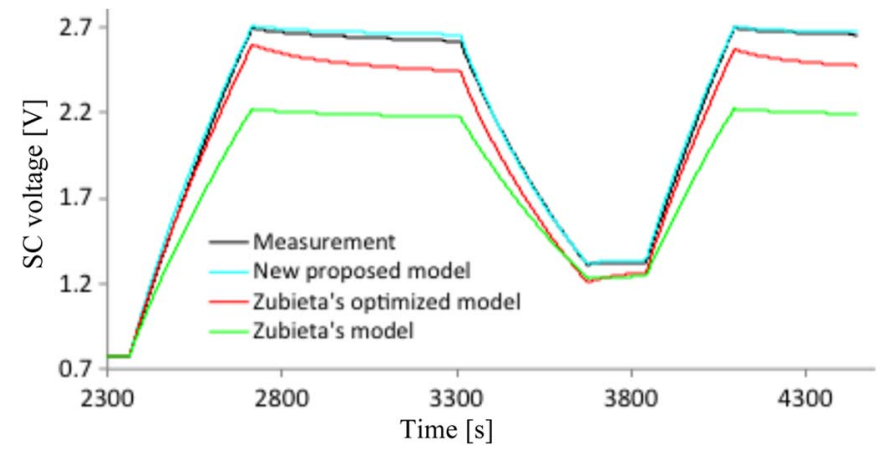

Fig. 11. Comparison between experimental results and simulation results (2-A constant charging current).

\section{A. Experimental Validation of Model During Dynamic Cycles}

In this paragraph, we will make reference to an SC cycle composed by the following parts: 1) Starting from a steady-state condition of the $\mathrm{SC}$ with a $V_{\mathrm{OC}}=0.77 \mathrm{~V}$, the $\mathrm{SC}$ is charged at a constant current of $2 \mathrm{~A}$ up to the maximum $\mathrm{SC}$ voltage (i.e., $2.7 \mathrm{~V}$ ) for $360 \mathrm{~s}$; 2) SC long resting phase of $600 \mathrm{~s}$ in order to let the redistribution phase taking place; 3) SC discharge with a constant resistance for $370 \mathrm{~s}$; 4) SC short resting phase of $170 \mathrm{~s}$ in order to avoid the presence of important redistribution phase; 5) SC charging at constant current up to the maximum SC voltage (i.e., 2.7 V), and, finally, 6) SC long resting phase of $400 \mathrm{~s}$. The combination of the aforementioned phases has been chosen in order to evaluate the SC behavior with a large number of operating conditions able to make visible the redistribution phenomenon.

Fig. 11 illustrates a first comparison among the measurement and SC model results with reference to the dynamic cycle described earlier. The simulation results refer to the optimized Zubieta's model [parameters calculated by (3) and (4)], the Zubieta's model [1], [2], and those obtained by using the model presented here (Fig. 4).

In what follows, we describe the assessment of the SC model parameters implemented into an automatic process. For the sake of clarity, the calculation of the parameters of the proposed SC model is detailed for each phase. In particular, the first one to be determined is $\Delta Q$ (associated to the current generator $I_{\mathrm{CH}}$ ). This parameter is inferred by using Fig. 6 for a $V_{\mathrm{OC}}=0.77 \mathrm{~V}$; the corresponding value is $\Delta Q=172 \mathrm{C}$.

During this charging phase (i), a certain amount of $\Delta Q$ is used because the generator $I_{\mathrm{CH}}$ remains active only if the SC supply is connected. In our case, such an amount is $\Delta Q_{1}=$ $51.2 \mathrm{C}$. At the beginning of phase (ii), the supply is turned off, and correspondingly, the SC redistribution phase takes place with a time constant on the order of $800 \mathrm{~s}$ (see Fig. 3).

By using the data of Table $\mathrm{I}$, it is possible to infer the value of $V_{\mathrm{OC}}$ that the $\mathrm{SC}$ will reach for an infinite time (in our case, this value is of $2.56 \mathrm{~V}$ ). With such a $V_{\mathrm{OC}}$ value, we can infer, from Fig. 10, the amount of the residual charge that the SC will have in case it will rest in open-circuit conditions. Such an amount $\Delta Q_{2}$ is equal to $38 \mathrm{C}$. During this phase, charges are naturally migrating from the different macropores toward the medium and micropores. This amount of charges has to be increased in 
comparison to the amount that would have been if the $\mathrm{SC}$ was totally discharged. At the beginning of this redistribution phase, the $\Delta Q_{\mathrm{RED}}$ is

$$
\Delta Q_{\mathrm{RED}}=\Delta Q-\Delta=(172-51.2) \mathrm{C}=120.8 \mathrm{C} .
$$

The quota of $\Delta Q_{\mathrm{RED}}$ contributing to the redistribution phase due to diffusion phenomena is, in view of the aforementioned consideration, given by

$$
\Delta Q_{\mathrm{RED}}^{*}=\Delta Q_{\mathrm{RED}}-\Delta Q_{2}=(120.8-38) \mathrm{C}=82.8 \mathrm{C} .
$$

This charge has to be redistributed by following (6).

The relevant time constants $\tau_{2}$ and $\tau_{3}$ are as follows: $\tau_{2}=$ $R_{2} C_{2}$ and $\tau_{3}=R_{3} C_{3}$, where $C_{2}$ and $C_{3}$ are the values of the nonlinear capacitances of the SC second and third branches, respectively (depending on $V_{F}$, see Tables IV and V).

This phase goes on for $600 \mathrm{~s}$ that is not enough for redistributing the whole $\Delta Q_{\mathrm{RED}}^{*}$. The effective redistributed quantity $\Delta Q_{3}=53 \mathrm{C}$ is calculated as follows:

$$
\Delta Q_{3}=\int \eta_{2} \frac{\Delta Q_{\mathrm{RED}}^{*}}{\tau_{2}} e^{-\frac{t}{\tau_{2}}}+\int \eta_{3} \frac{\Delta Q_{\mathrm{RED}}^{*}}{\tau_{3}} e^{-\frac{t}{\tau_{3}}} .
$$

The charges available for the following discharging phase are equal to:

$\Delta Q_{\mathrm{DISCH}}=\Delta_{\mathrm{RED}}^{*}-\Delta Q_{3}+\Delta Q_{2}=82.853+38=67.8 \mathrm{C}$.

This charge has to be redistributed with a time constant equal to $1000 \mathrm{~s}$ (this value depend on the reached voltage value, and it has been experimentally assessed). During this phase, a quota of $\Delta Q_{\mathrm{DISCH}}$, named $\Delta Q_{4}$, is used (charges coming from micro- and medium pores migrate toward the macropores), and it is still available inside the SC: It increases the quantity of the available charge for the next phase. At the beginning of the following redistribution phase, the available charge $\Delta Q_{\mathrm{RED}, 2}$ is

$$
\Delta Q_{\mathrm{RED}, 2}=\Delta Q_{\mathrm{DISCH}}+\Delta Q_{4}=67.8+55.2=123 \mathrm{C} .
$$

The whole redistribution phase always takes a long time, already detailed on the order of $3000 \mathrm{~s}$, and after this time, the $\Delta Q_{\mathrm{END}, 2}$ will be equal to $161.1 \mathrm{C}$ (value taken from Fig. 10). In this respect, the charge gradient equals

$$
\Delta Q_{\mathrm{RED}, 2}^{*}=\Delta Q_{\mathrm{RED}, 2}-\Delta Q_{\mathrm{END}, 2}=123-161.1+-38.1 \mathrm{C} .
$$

The negative value means that these charges are not migrated from micro-medium pores toward large pores but in the opposite way. For this reason, the sign of $I_{\mathrm{RED}}$ should be negative; this current increases the total amount of charges stored in the micro-medium pores. If this phase takes enough time (3000 s) at its end, the available charge should be $\Delta Q_{\mathrm{END}, 2}$. Since, in our dynamic cycle, this phase lasted for $170 \mathrm{~s}$, the quota of redistributed $\Delta Q_{\mathrm{RED}, 2}^{*}$, named $\Delta Q_{5}$, can be calculated as follows:

$$
\Delta Q_{5}=\int \eta_{2} \frac{\Delta Q_{\mathrm{RED}}}{\tau_{2}} e^{-\frac{t}{\tau}}-\int \eta_{3} \frac{\Delta Q_{\mathrm{RED}}^{*}}{\tau_{3}} e^{\frac{t}{t_{3}}}=14.1 \mathrm{C} .
$$

The available charge $\Delta Q_{6}$ for the next charge phase will be

$$
\begin{aligned}
\Delta Q_{6} & =\Delta Q_{\mathrm{END}, 2}+\Delta Q_{\mathrm{RED}, 2}^{*}+\Delta Q_{5} \\
& =161.138 .1+14.1=137.1 \mathrm{C} .
\end{aligned}
$$

At the beginning of the last charging phase, the generator $I_{\mathrm{CH}}$ should be turned on and the following additional virtual current should be delivered:

$$
\frac{\Delta Q_{6}}{\tau} e^{-\frac{t}{\tau}}=\frac{137.1}{1000} e^{-\frac{t}{\tau}} A=0.137 e^{-\frac{t}{\tau}} A .
$$

During this charging phase, a quota of $\Delta Q_{6}$, named $\Delta Q_{7}$ and equal to $33 \mathrm{C}$, is used and stored inside the $\mathrm{SC}$.

The available charge at the beginning of the last redistribution phase is $\Delta Q_{\mathrm{RED}, 3}$

$$
\Delta Q_{\mathrm{RED}, 3}=\Delta Q_{\mathrm{START}, 2}-\Delta Q_{7}=137-33=104 \mathrm{C} .
$$

The quota of $\Delta Q_{\mathrm{RED}, 3}$ contributing to the last redistribution phase due to diffusion phenomena of the charges equals

$$
\Delta Q_{\mathrm{RED}, 3}=\Delta Q_{\mathrm{RED}, 3}-\Delta Q_{2}=104-38 \mathrm{C}=66.1 \mathrm{C} .
$$

Since the reached voltage after the charging phase is the same, the two time constants for this redistribution phase are the same of those calculated in (12).

It should be underlined that the error between the SCmeasured voltage and the predicted one is extremely low. In particular, a mean-squared error of $6.85 \cdot 10^{-4}$ has been estimated for the model proposed here and a corresponding value of 0.124 has been evaluated for the Zubieta's one.

In the comparison shown in Fig. 11, the current has the same value as the one used to infer the SC parameters (see Fig. 5).

Then, in order to better underline the improvement provided by the proposed model, in what follows, we present further comparisons between measurements and model predictions with reference to these conditions: 1) charging current different from the one used to asses the SC parameters; 2) short and long redistribution phases; and 3) different values of open-circuit starting voltages. To the best of the authors' knowledge, the validation of an SC model with respect to all these extensive analyses has not been performed by available SC models.

In this respect, Fig. 12 shows the results for an SC cycle with a lower value of the charging current equal to $1.5 \mathrm{~A}$. We can notice the same accuracy obtained for the previous case. Fig. 13 refers to a cycle with repetitive charging/ discharging/redistribution phases. This specific cycle has been chosen since SCs are often subjected to this type of stresses. The value of the charging current is equal to $2.3 \mathrm{~A}$. Fig. 14 shows the comparison for a cycle characterized by a deep discharging phase (from the nominal value until $0.5 \mathrm{~V}$ ) that represents a challenge for all the previous SC models. The difference between the measurement and voltage value predicted 


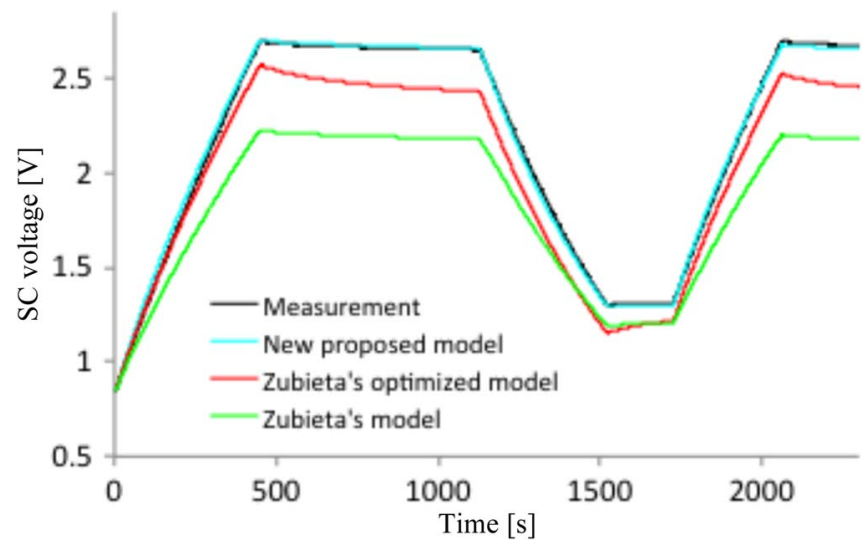

Fig. 12. Comparison between experimental results and simulation results (1.5-A constant charging current).

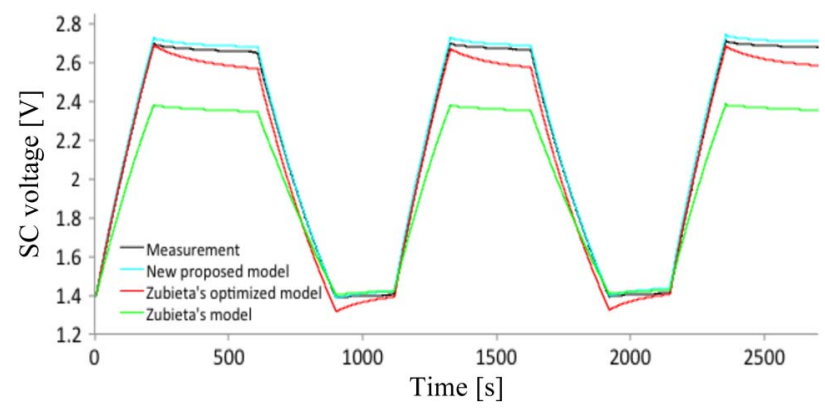

Fig. 13. Comparison between experimental results and simulation results (2.3-A constant charging current).

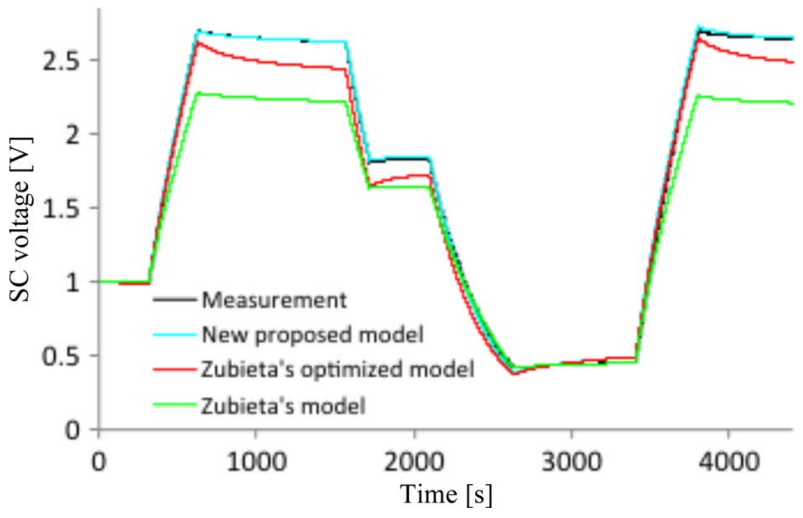

Fig. 14. Comparison between experimental results and simulation results (2-A constant charging current and deep discharging phase).

by the Zubieta's model is around $15 \%$ instead of the $0.2 \%$ reached with the model proposed here.

Finally, Fig. 15 illustrates the comparison for an SC cycle with two short charging phases and two short discharging phases followed by a long rest phase. We can observed that the model presented here is able to follow the right value with higher precision even if the redistribution of charges does not take place.

\section{CONCLUSION}

This paper has first discussed the experimental evidences related to charge-redistribution phenomenon that takes place in SCs. As known, this phenomenon is associated to the diffusion

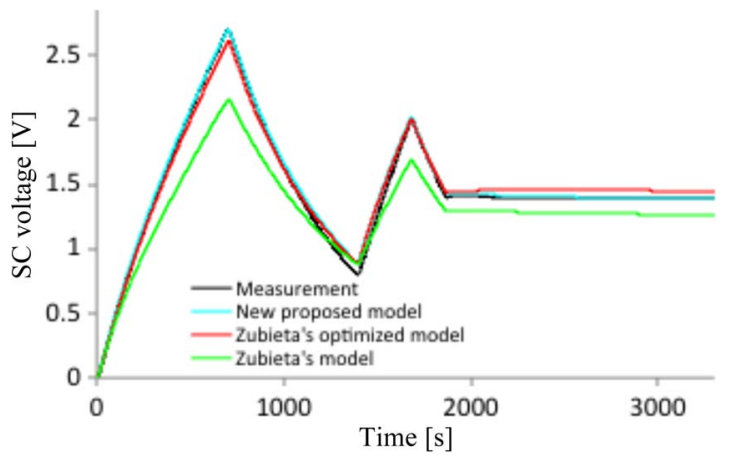

Fig. 15. Comparison between experimental results and simulation results (only one long rest phase).

of the SC residual charges during charging/discharging and rest phases. As it has a large influence on the SC dynamic behavior, it deserves a specific modeling in order properly predict the SC voltage dynamic. In this respect, a first discussion about the improvement of the most common SC model has been included in this paper. The proposed improvement consists in the optimal assessment of the SC model parameters by means of a least square technique. Then, this paper has proposed a new SC model where specific current sources, specifically dealing with the representation of the SC diffusion of residual charges, have been included.

The experimental procedure to evaluate these current source parameters has been also presented and discussed. The experimental validation of the proposed SC model with reference to different dynamic cycles has been finally included and discussed to show the robustness of the proposed model.

It is finally worth observing that, compared to the existing SC models, the one proposed here allows for reducing the meansquare errors between measurements and simulation results of three orders of magnitude.

\section{ACKNOWLEDGMENT}

The authors would like to thank the EOS-Holding and the Swiss Innovation and Technology Committee (CTI) as the results shown in this paper have been partially obtained within ongoing research activities sponsored by these entities and the Distributed Electrical Systems Laboratory, Swiss Federal Institute of Technology (EPFL).

\section{REFERENCES}

[1] F. Belhachemi, S. Rael, and B. Davat, "A physical based model of power electric double-layer supercapacitors," in Conf. Rec. IEEE IAS Annu. Meeting, 2000, pp. 3069-3076.

[2] L. Zubieta and R. Bonert, "Characterization of double-layer capacitors for power electronics applications," IEEE Trans. Ind. Appl., vol. 36, no. 1, pp. 199-205, Jan./Feb. 2000.

[3] N. Bertrand, J. Sabatier, O. Briat, and J. Vinassa, "Embedded fractional nonlinear supercapacitor model and its parametric estimation method," IEEE Trans. Ind. Electron., vol. 57, no. 12, pp. 3991-4000, Dec. 2010.

[4] A. S. Weddell, G. V. Merrett, T. J. Kazmierski, and B. M. Al-Hashimi, "Accurate supercapacitor modeling for energy harvesting wireless sensor nodes," IEEE Trans. Circuits Syst. II, Exp. Briefs, vol. 58, no. 12, pp. 911915, Dec. 2011.

[5] V. Musolino, L. Piegari, and E. Tironi, "New full frequency range supercapacitor model with easy identification procedure," IEEE Trans. Ind. Electron., vol. 60, no. 1, pp. 112-120, Jan. 2013. 
[6] F. Rafik, H. Gualous, R. Gallay, A. Crausaz, and A. Berthon, "Frequency, thermal and voltage supercapacitor characterization and modeling," J. Power Sources, vol. 165, no. 2, pp. 928-934, Mar. 2007.

[7] N. Rizoug, P. Bartholomeus, and P. Le Moigne, "Modeling and characterizing supercapacitors using an online method," IEEE Trans. Ind. Electron., vol. 57, no. 12, pp. 3980-3990, Dec. 2010.

[8] S. Buller, E. Karden, D. Kok, and R. W. De Doncker, "Modeling the dynamic behavior of supercapacitors using impedance spectroscopy," in Conf. Rec. IEEE 36th IAS Annu. Meeting, Sep. 2001, vol. 4, pp. 2500-2504.

[9] S. H. Kim, W. Choi, K. B. Lee, and S. Choi, "Advanced dynamic simulation of supercapacitors considering parameter variation and selfdischarge," IEEE Trans. Power Electron., vol. 26, no. 11, pp. 3377-3385, Nov. 2011.

[10] J. H. Chang, F. P. Dawson, and K. Lian, "A first principles approach to develop a dynamic model of electrochemical capacitors," in Proc. IPEC, Jun. 21-24, 2010, pp. 2382-2389.

[11] M. Xiao, S.-Y. Choe, and F. Rahman, "Static and dynamic analysis of Li-polymer battery using thermal electrochemical model," in Proc. IEEE CITRES, Sep. 27-29, 2010, pp. 309-316.

[12] M. Kaus, J. Kowal, and D. U. Sauer, "Modeling the effects of charge redistribution during self-discharge of supercapacitors," Electrochim. Acta J., vol. 55, no. 25, pp. 7516-7523, Oct. 2010.

[13] R. Lu, G. Wu, R. Ma, and C. Zhu, "Model based state of charge estimation method for ultra-capacitor," in Proc. IEEE Veh. Power Propulsion Conf., Sep. 2008, pp. 1-5.

[14] L. Zhongxue and C. Jie, "An impedance-based approach to predict the state of charge for carbon-based supercapacitors," Microelectron. Eng. J., vol. 85, no. 7, pp. 1549-1554, Jul. 2008.

[15] B. W. Ricketts and C. Ton-That, "Self-discharge of carbon-based supercapacitors with organic electrolytes," J. Power Sources, vol. 89, no. 1, pp. 64-69, Jul. 2000.

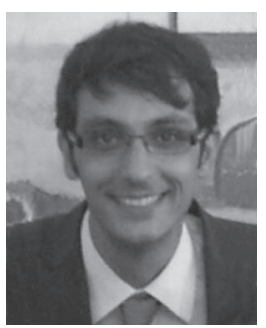

Dimitri Torregrossa was born in Palermo, Italy, in 1982. He received the M.S. degree from the University of Studies of Palermo, Palermo, in 2007 and the Ph.D. degree from the University of Technology of Belfort-Montbéliard (UTBM), Belfort, France, in 2010 .

From 2010 to 2011, he was a Lecturer of power systems and electric drives with UTBM. In 2012, he joined as a Researcher the Distributed Electrical Systems Laboratory headed by Prof. Paolone with the Swiss Federal Institute of Technology (EPFL), Lausanne, Switzerland. He is the author or coauthor of more than 15 scientific papers published in reviewed journals and presented at international conferences. His special interests include dynamic electric behavior and ageing model of energy-storage systems, design of microgrid, mechanical-acoustic, and electromagnetic finite-element analysis.

Dr. Torregrossa was the recipient of the Associate Professor eligibility from NCU, France, in 2010.

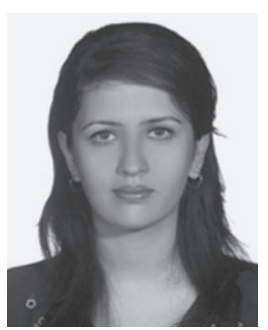

Maryam Bahramipanah was born in Tehran, Iran, in 1984. She received the M.S. degree in electrical engineering from the University of Tehran, Tehran, in 2011. She is currently working toward the Ph.D. degree in power system engineering at the Swiss Federal Institute of Technology (EPFL), Lausanne, Switzerland.

Her research interests include renewable energy system, energy-storage systems, and power system control.

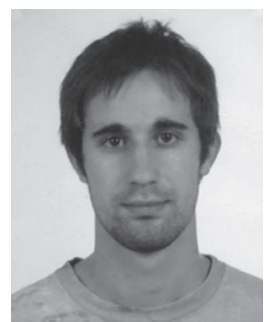

Emil Namor was born in Cividale del Friuli, Italy, in 1989. He received the B.Sc. degree in energy engineering from the University of Padova, Padova, Italy, in 2012, with an experimental thesis on the circuit models of supercapacitors, developed at the Distributed Electrical Systems Laboratory, Swiss Federal Institute of Technology (EPFL), Lausanne, Switzerland, and also from the École Centrale de Lille, Villeneuve d'Ascq, France, within the doubledegree program TIME. He is currently working toward the M.Sc. degree in electrical engineering at the

University of Padova.

His main fields of interest are electrical systems, energy storage, and electromobility.

Mr. Namor is a CIGRE young member.

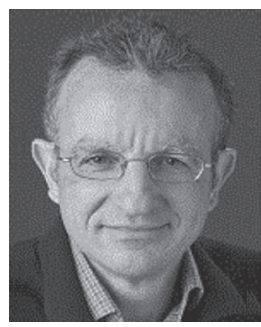

Rachid Cherkaoui (M'05-SM'07) received the M.Sc. and Ph.D. degrees in electrical engineering from the Swiss Federal Institute of Technology in Lausanne (EPFL), Lausanne, Switzerland, in 1983 and 1992, respectively.

$\mathrm{He}$ is currently a Senior Scientist with EPFL, leading the power systems group. He is the author and coauthor of more than 80 scientific publications. His research interests are in electricity market deregulation, distributed generation and storage, and power system vulnerability mitigation.

Dr. Cherkaoui is a Member of the Technical Program Committees of various conferences, a member of CIGRE Task Forces and working groups. He was an IEEE Swiss Chapter Officer from 2005 to 2011.

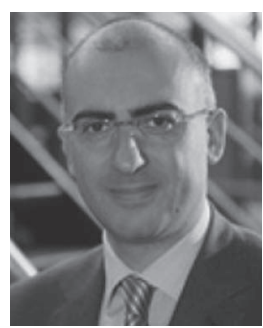

Mario Paolone (M'07-SM'10) was born in Italy in 1973. He received the M.Sc. (Hons.) and Ph.D. degrees in electrical engineering from the University of Bologna, Bologna, Italy, in 1998 and 2002, respectively.

In 2005, he was appointed as a Researcher in electric power systems with the University of Bologna where he was with the Power Systems Laboratory until 2011. He is currently an Associate Professor with the Swiss Federal Institute of Technology of Lausanne (EPFL), Lausanne, Switzerland, where he accepted the EOS Holding Chair of Distributed Electrical Systems Laboratory. $\mathrm{He}$ is the author or coauthor of more than 160 scientific papers published in reviewed journals and presented at international conferences. His research interests are in the areas of smart grids, with particular reference to real-time monitoring and operation, power system protections, power system dynamics, and power system transients with particular reference to LEMP interaction with electrical networks.

Dr. Paolone is a Secretary and a member of several IEEE and CIGRÉ working groups. He was Cochairperson of the Technical Committee of the ninth edition of the International Conference of Power Systems Transients. In 2010, he was the recipient of the Associate Professor eligibility from the Politecnico di Milano, Milan, Italy. In 2013, he is the recipient of the IEEE EMC Technical Achievement Award. 